Violeta Rodrigues Aguiar ${ }^{1}$ Roger Keller Celeste ${ }^{1}$

\section{Necessidade e alocação de laboratórios regionais de prótese dentária no Brasil: um estudo exploratório}

\author{
The need for, and allocation of, regional prosthodontics \\ laboratories in Brazil: an exploratory study
}

'Departamento de Odontologia Preventiva e Social, Faculdade de Odontologia, Universidade Federal do Rio Grande do Sul. R. Ramiro Barcelos 2492, Santana. 90035-003 Porto Alegre RS Brasil. violetaaguiar@gmail.com
Abstract The scope of this study was to compare epidemiological indicators of the need for prosthetic rehabilitation in 2003 with the number of regional prosthodontic laboratories (LRPD) and technicians in prosthodontics (TPD) in the five Brazilian regions between 2012 and 2013. Data regarding health services were obtained from DATASUS and epidemiological data were $o b$ tained from SBBrasil 2003. The rates of dental prostheses produced and LRPD and TPD were calculated per 100,000 people. The Kruskal-Wallis and chi-square test were used for analysis. The results show that the sex/age adjusted prevalence of edentulism varied between regions $(p<0.01)$, being higher in the Southeast (13.9\%). The highest mean number of missing teeth was found in the North (10.3 teeth). The highest availability of LRPD (1.16 per 100,000) and TPD (1.05 per 100,000) occurred in the Northeast region. The highest need for complete dentures (7.2\%) was reported in the North, but was the lowest in delivery of dentures (9.71 per 100,000). There were statistically significant differences $(p<0.01)$ among regions for both LRPD, TPD rates for prostheses. It is considered that criteria other than epidemiological aspects influenced the opening of LRPD in the country.

Key words Unified Health System, Health services evaluation, Health information systems, Oral health, Health surveys
Resumo Objetivou-se comparar os indicadores epidemiológicos e de necessidade de reabilitação protética de 2003 com o número de Laboratórios Regionais de Prótese Dentária (LRPD) e Técnicos em Prótese Dentária (TPD), nas cinco regiões brasileiras, entre 2012 e 2013. Os dados sobre os serviços de saúde foram obtidos no DATASUS e os epidemiológicos a partir SBBrasil 2003. As taxas de próteses dentárias produzidas, de LRPD e de TPD foram calculadas por 100.000 habitantes. Para a análise, utilizaram-se os testes de Kruskal - Wallis e Qui-quadrado. A prevalência de edentulismo, ajustada por sexo e idade variaram entre as regiões ( $p<0,01)$, sendo maior na região Sudeste (13,9\%). O maior número médio de dentes perdidos foi encontrado na região Norte (10,3 dentes). A maior disponibilidade de LRPD (1,16 por 100 mil/hab) e TPD (1,05 por 100 mil/hab) ocorreu na região Nordeste. A maior necessidade de prótese total (7,2\%) foi registrada no Norte, mas a mesma foi a mais baixa na entrega de próteses (9,71 por $100 \mathrm{mil} / \mathrm{hab})$. Houve diferença estatisticamente significativa $(p<0,01)$ entre as regiões, para taxas de LRPD, TPD e de próteses. Sugere-se que outros critérios além dos epidemiológicos, influenciaram na abertura de LRPD no país.

Palavras-chave Sistema Único de Saúde, Avaliação de Serviços de Saúde, Sistemas de Informação em Saúde, Saúde Bucal, Levantamento Epidemiológico 
Introdução

Segundo a Organização Mundial da Saúde (OMS), em 2010, o edentulismo representava a $3^{\circ}$ condição de saúde bucal com maior Carga Global das Doenças ${ }^{1}$. Esse agravo é caracterizado como a sequela de maior gravidade da cárie dentária e doenças periodontais ${ }^{2}$, e está diretamente relacionado a problemas de mastigação, dor, dificuldade de alimentação e de relacionamento social $^{3,4}$, principalmente nos idosos, impactando, diretamente, na qualidade de vida das pessoas ${ }^{5,6}$.

De acordo com os resultados do último inquérito nacional ${ }^{7}$ percebe-se que a necessidade de reabilitação protética aumenta com o avanço da idade. Entre 15 e 24 anos de idade, 13,7\% dos indivíduos necessitam de prótese dentária, de 25 a 44 anos, $68,8 \%$, e idosos de 65 a 74 anos, 92,7\%. Revela-se também alta prevalência de edentulismo $(9 \%)$ e elevado índice CPOD médio $(20,4)$ na população brasileira entre 35 e 44 anos de ida$\mathrm{de}^{8}$, demonstrando a grande necessidade de tratamento ainda existente, e que recai no sistema público de saúde do país 9 .

Em função dessa realidade, o Ministério da Saúde (MS), pautado pela Política Nacional de Saúde Bucal ${ }^{10}$, tem aumentado a oferta de serviços de reabilitação protética dentária e a capacitação de Técnicos em Prótese Dentária (TPD) para o Sistema Único de Saúde (SUS). O acréscimo no financiamento aos serviços públicos de atenção especializada, através das portarias $n^{\circ}$ $211^{11}, \mathrm{n}^{\circ} 2.374^{12}$ e $^{\circ} 1.895^{13}$, favoreceu a ampliação do acesso ${ }^{7}$. De acordo com a Sala de Apoio à Gestão Estratégica, de 2005 a 2013 ${ }^{14}, 1465$ Laboratórios de Prótese Dentária (LRPD) foram habilitados no país.

Reconhece-se que a decisão sobre a alocação de estabelecimentos e a adesão a programas de saúde pode ser guiada por múltiplas questões, como políticas e financeiras ${ }^{15-18}$. Entretanto, a legislação do SUS enfatiza a importância da utilização da epidemiologia ${ }^{19-21}$ como norteadora dos processos de planejamento em saúde. Dessa forma, poder-se-ia esperar que, em nível nacional, macrorregiões geográficas com piores indicadores de saúde bucal no inquérito epidemiológico de 2003 receberiam maior aporte de recursos e infraestrutura.

Assim, o objetivo deste estudo é comparar os indicadores epidemiológicos de prevalência de edentulismo, de dentes perdidos e de necessidade de reabilitação protética do inquérito de 2003 com a distribuição do número de LRPD e de TPD nas 5 macrorregiões brasileiras, de 2012 a 2013.

\section{Metodologia}

\section{Delineamento e fonte de dados}

Este é um estudo misto, ecológico (dados de serviços de saúde) e individual (dados epidemiológicos). Para os dados ecológicos, o nível de agregação foram as cinco macrorregiões brasileiras. As informações sobre a produção ambulatorial (quantidade apresentada) foram coletadas para os meses de janeiro a dezembro dos anos de 2012 e 2013 no site do Departamento de Informática do Ministério da Saúde (Datasus), em maio de 2014. Utilizaram-se dados do Cadastro Nacional de Estabelecimentos de Saúde (CNES) para informações sobre LRPD credenciados e número de TPD que atendem no SUS, dados do Sistema de Informações Ambulatoriais (SIA-SUS) para produção de próteses; e dados do IBGE para informações demográficas. Em nível individual, foi utilizado dados do SBBrasil $2003^{22}$ para as faixas etárias de jovens (15-19 anos), adultos (34-45 anos) e idosos (65-74 anos).

\section{Variáveis epidemiológicas \\ e de necessidade de prótese}

Foram utilizados dois indicadores epidemiológicos e três de necessidade de prótese dentária. Os indicadores epidemiológicos derivam do exame clínico odontológico da coroa dentária: prevalência de edentulismo e média de dentes perdidos. Para o edentulismo foi considerado a perda de 32 dentes por qualquer motivo. Considerou-se perda dental a ausência do elemento dentário segundo os códigos 4 (perdido por cárie) ou 5 (perdido por outro motivo). Os indicadores de necessidade foram obtidos a partir do exame clínico de necessidade de prótese: prevalência de prótese total (código 4), prevalência de prótese parcial de 2 ou mais elementos dentários (códigos 2 e 3 ) e prevalência de prótese fixa ou removível para 1 elemento dentário (código 1). Foi considerada a necessidade de prótese na arcada superior ou inferior.

Para fins de comparabilidade entre as macrorregiões, foram calculados valores de prevalência ajustados, utilizando-se o método direto de padronização ${ }^{23}$ que teve a população total do inquérito SB Brasil 2003 como referência de cálculo para faixa etária e sexo. Assim, as prevalências ajustadas refletem a prevalência que cada macrorregião teria se todas tivessem uma população com a mesma estrutura de sexo e idade.

Foram utilizados dados do SB Brasil de 2003, anteriores à implantação da política de laborató- 
rios de prótese, pois se reconhece que o uso dos dados de 2010 implicaria em avaliação da política, e não em informação para a tomada de decisão.

\section{Variáveis relacionadas a serviços de saúde}

Os códigos de produção ambulatorial escolhidos foram aqueles relacionados ao monitoramento da produção ambulatorial proposta pela Nota Técnica ${ }^{24}$ da Coordenação Geral de Saúde Bucal do Ministério da Saúde. Obtiveram-se as taxas mensais de próteses totais (PT) a partir dos códigos 0701070129 para prótese total mandibular e 0701070137 para prótese total maxilar; taxas mensais de próteses fixas (PF) a partir do código 0701070145 para próteses coronárias/intrarradiculares fixas/adesivas (por elemento); taxas mensais de próteses parciais removíveis (PPR) a partir dos códigos 0701070099 para prótese parcial mandibular removível e 0701070102 para prótese parcial maxilar removível.

As demais variáveis relativas aos serviços de saúde foram taxas mensais de LRPD e de TPD. Para todas as taxas considerou-se a produção dos 24 meses, ou seja, de janeiro de 2012 a dezembro 2013. O cálculo de taxas (por 100 mil habitantes/mês) foi realizado utilizando-se a quantidade aprovada para cada mês de cada macrorregião específica, sendo o denominador a população acima de 15 anos de idade, estimada para 2012, segundo o IBGE.

As produções ambulatoriais e o quantitativo de TPD e LRPD credenciados disponíveis ao SUS foram organizados em duas categorias, considerando o tipo de prestador. A primeira refere-se a todos os tipos de serviço (público, privado, sindicato, filantrópico) e a segunda somente àquela de caráter público.

\section{Análise estatística}

Variáveis binárias foram descritas na forma de percentuais com intervalos de confiança de 95\% e taxas por meio de médias e desvios-padrão em tabelas. Diferenças entre as 5 macrorregiões foram testadas com o teste Qui-quadrado para heterogeneidade para variáveis categóricas e com o teste não paramétrico Kruskal-Wallis para taxas. A análise estatística foi realizada no software $\mathrm{R}$.

\section{Aspectos éticos}

O SB Brasil 2003 foi submetido ao Comitê de Ética em Pesquisa do Ministério da Saúde e aprovado.

\section{Resultados}

No inquérito epidemiológico o tamanho da amostra foi de 35.613 indivíduos nas faixas etárias consideradas, porém 1,4\% $(\mathrm{n}=484)$ da amostra possuía dados faltantes nas variáveis de perda dental ou necessidade de prótese. A região com maior percentual de dados faltantes foi a região Sudeste (2,3\%). Verificou-se que nos meses de outubro a dezembro de 2012, não existiam, no SIA-SUS, informações sobre o quantitativo total de LRPD de cada macrorregião, assim as análises dessas taxas referem-se a 21 meses (3meses faltantes).

A prevalência ajustada de edentulismo variou entre as regiões $(\mathrm{p}<0,01$, Qui-quadrado), sendo a maior na Sudeste (13,9\%), e a menor no Nordeste (10,5\%) (Tabela 1). O número médio de dentes perdidos também apresentou variação entre as macrorregiões ( $\mathrm{p}<0.01$, Qui-quadrado) e a maior média foi encontrada na região Norte (10,3 dentes) e o menor na região Sudeste $(8,7$ dentes). A maior necessidade de PT foi observada na região Norte $(7,2 \%)$ e a menor na Sul (3,9\%). Quanto à necessidade de PPR, a maior prevalência foi encontrada na região Nordeste $(42,1 \%)$ e a menor $(28,8 \%)$ na Sudeste. Já no que se refere à prótese fixa (coroa ou faceta), a maior necessidade de tratamento foi verificada no Centro-Oeste $(2,9 \%)$ e a menor no Norte $(1,0 \%)$.

Houve diferenças estatisticamente significativas ( $\mathrm{p}<0,01$, Kruskal-Wallis) entre as macrorregiões tanto para as taxas de LRPD, taxa de TPD, quanto para taxa de próteses totais, de próteses parciais e de próteses fixas. A maior taxa de LRPD por 100.000 habitantes-mês foi encontrada na região Nordeste (Tabela 2), com 1,16 (DP+/-0,34) e a menor na Norte com $0,51(\mathrm{DP}+/-0,11)$. Da mesma forma, a taxa de TPD foi maior nas regiões Nordeste e Centro-oeste, ambas com 1,05 (DP+/-0,10), e a menor no Norte de 0,54 (DP+/$0,05)$. Verificou-se que a taxa de PT e PPR por 100.000 habitantes/mês foi maior na Nordeste com, respectivamente, 20,75 (DP+/-6,12) e 9,14 (DP+/-3,61). Já a menor em ambas foi na Norte com 9,71 PT (DP+/-1,38) e 4,27 (DP+/$0,63)$ PPR por 100.000 habitantes/mês. Como as demais, a menor oferta de PF por 100.000 habitantes/mês ocorreu na região Norte com 0,03 (DP+/-0,03) e a maior utilização ocorreu na Sudeste com 1,65 (DP+/-0,45).

As diferenças regionais nos componentes de oferta e utilização aumentaram ao considerarmos somente aqueles prestadores de serviços de caráter público. Avaliando-se a distribuição dos pres- 
Tabela 1. Indicadores epidemiológicos de saúde bucal e necessidade normativa de tratamento protético para população brasileira com intervalos de confiança de 95\% (IC95\%), por macrorregião. Brasil, 2003.

\begin{tabular}{|c|c|c|c|c|c|c|c|}
\hline \multicolumn{2}{|c|}{ Macrorregião } & \multicolumn{2}{|c|}{ Pessoas com Edentulismo } & \multicolumn{2}{|c|}{ Dentes Perdidos } & \multicolumn{2}{|c|}{$\begin{array}{c}\text { Pessoas com Necessidade } \\
\text { de Prótese Total (Inferior } \\
\text { ou Superior) }\end{array}$} \\
\hline & $\mathbf{n}$ & $\%$ Ajustada & (IC95\%) & Média Ajustada & (IC95\%) & $\%$ Ajustada & (IC95\%) \\
\hline $\mathrm{N}$ & 6.990 & 11,4 & $(10,7-12,0)$ & 10,3 & $(10,2-10,3)$ & 7,2 & $(6,5-7,8)$ \\
\hline $\mathrm{NE}$ & 8.693 & 10,5 & $(10,0-11,0)$ & 9,6 & $(9,6-9,7)$ & 6,4 & $(5,9-6,8)$ \\
\hline SE & 6.224 & 13,9 & $(13,3-14,5)$ & 8,7 & $(8,6-8,7)$ & 6,9 & $(6,4-7,5)$ \\
\hline S & 8.360 & 11,0 & $(10,5-11,5)$ & 8,8 & $(8,8-8,9)$ & 3,9 & $(3,5-4,2)$ \\
\hline $\mathrm{CO}$ & 4.862 & 12,2 & $(11,5-12,9)$ & 9,0 & $(8,9-9,0)$ & 5,7 & $(5,2-6,3)$ \\
\hline \multicolumn{2}{|c|}{ p-valor ${ }^{*}$} & $<0,01$ & & $<0,01$ & & $<0,01$ & \\
\hline \multirow[t]{2}{*}{ Total } & 35.129 & 11,8 & $(11,2-12,4)$ & 9,27 & $(9,2-9,3)$ & 6,0 & $(5,5-6,5)$ \\
\hline & Macrorregião & \multicolumn{3}{|c|}{$\begin{array}{l}\text { Pessoas com Necessidade de } \\
\text { Prótese de } 2 \text { ou mais dentes }\end{array}$} & \multicolumn{3}{|c|}{$\begin{array}{l}\text { Pessoas com Necessidade de } \\
\text { Prótese de } 1 \text { dente }\end{array}$} \\
\hline & $\mathrm{N}$ & \multicolumn{2}{|c|}{$\%$ Ajustada } & (IC95\%) & $\%$ Ajustada & \multicolumn{2}{|l|}{ (IC95\%) } \\
\hline $\mathrm{N}$ & 6.990 & & 41,2 & $(40,2-42,8)$ & 1,0 & $(0,7-1,2)$ & \\
\hline $\mathrm{NE}$ & 8.693 & & 42,1 & $(41,3-43,0)$ & 1,4 & $(1,2-1,6)$ & \\
\hline SE & 6.224 & & 28,8 & $(27,9-29,8)$ & 2,6 & $(2,2-3,0)$ & \\
\hline S & 8.360 & & 30,8 & $(29,9-31,7)$ & 1,6 & $(1,3-1,9)$ & \\
\hline $\mathrm{CO}$ & 4.862 & & 34,4 & $(33,2-35,5)$ & 2,9 & $(2,5-3,4)$ & \\
\hline \multicolumn{2}{|c|}{ p-valor } & & $<0,01$ & & $<0,01$ & & \\
\hline Total & 35.129 & & 44,1 & $(43,1-45,1)$ & 1,9 & $(1,6-2,2)$ & \\
\hline
\end{tabular}

Nota: Valores ajustados pelo método direto de padronização para a população total do inquérito SB Brasil 2003 (15-19,35-44,65-74)

${ }^{*}$ Teste de qui-quadrado para heterogeneidade entre as macrorregiões.

Tabela 2. Médias mensais e desvios-padrão (DP) de indicadores de oferta e utilização relacionados à produção de próteses dentárias no Sistema Único de Saúde por macrorregião na população com mais de 15 anos de idade. Brasil, 2012-2013.

\begin{tabular}{|c|c|c|c|c|c|c|c|c|c|c|}
\hline \multirow[t]{2}{*}{ Macrorregião } & \multicolumn{2}{|c|}{$\begin{array}{c}\text { LRPD }^{* *} / 100 \mathrm{mil} \\
\text { hab-mês }\end{array}$} & \multicolumn{2}{|c|}{$\begin{array}{c}\mathrm{TPD}^{* * *} / 100 \mathrm{mil} \\
\text { hab-mês }\end{array}$} & \multicolumn{2}{|c|}{$\begin{array}{c}\text { Taxa de próteses } \\
\text { totais/100mil } \\
\text { hab-mês }\end{array}$} & \multicolumn{2}{|c|}{$\begin{array}{c}\text { Taxa de próteses } \\
\text { parciais } \\
\text { removíveis/100mil } \\
\text { hab-mês }\end{array}$} & \multicolumn{2}{|c|}{$\begin{array}{c}\text { Taxa de próteses } \\
\text { fixas/100mil } \\
\text { hab-mês }\end{array}$} \\
\hline & Média & $(\mathrm{DP}+/-)$ & Média & $(\mathrm{DP}+/-)$ & Média & $(\mathrm{DP}+/-)$ & Média & $(\mathrm{DP}+/-)$ & Média & $(\mathrm{DP}+/-)$ \\
\hline $\mathrm{N}$ & 0,51 & $(0,11)$ & 0,54 & $(0,05)$ & 9,71 & $(1,38)$ & 4,27 & $(0,63)$ & 0,03 & $(0,03)$ \\
\hline $\mathrm{NE}$ & 1,16 & $(0,34)$ & 1,05 & $(0,11)$ & 20,75 & $(6,1$ & 9,14 & $(3,6$ & 0,71 & $(0,23)$ \\
\hline SE & 0,75 & $(0,16)$ & 0,83 & $(0,06)$ & 15,47 & $(2,7$ & 5,07 & (1, & 1,65 & $(0,4$ \\
\hline S & 0,86 & $(0,18$ & 0,87 & $(0,08$ & 18,44 & $(3,9$ & 7,24 & $(2,04)$ & 0,26 & $(0,1$ \\
\hline $\mathrm{CO}$ & 0,83 & $(0,23)$ & 1,05 & $(0,10)$ & 14,69 & $(3,64)$ & 5,80 & $(2,10)$ & 1,06 & $(0,66)$ \\
\hline p-valor ${ }^{*}$ & $<0,01$ & & $<0,01$ & & $<0,01$ & & $<0,01$ & & $<0,01$ & \\
\hline Total & 0,82 & $(0,20)$ & 0,87 & $(0,08)$ & 15,81 & $(3,56)$ & 6,30 & $(1,91)$ & 0,74 & $(0,30)$ \\
\hline
\end{tabular}

* teste Kruskal-Wallis para diferenças entre macrorregiões; ${ }^{* *}$ LRPD: laboratório regional de prótese dentária; ${ }^{* *}$ TPD: Técnico de Prótese Dentária.

tadores de serviços constatou-se que a macrorregião Norte é constituída pela maior parcela pública, apresentando cerca $96,9 \%$ de suas próteses produzidas originalmente nesses estabelecimen- tos, ao passo que na Sul, $67,3 \%$ dos seus LRPD e $52,5 \%$ dos seus TPD provêm de estabelecimentos de caráter não públicos (filantrópicos, sindicatos e/ou privados) que fornecem serviços para o SUS. 
Período analisado.

Por fim, a ampliação no número dos LRPD no país e a produção parecem discretas, tendo em visa a demanda por reabilitação protética.

Diante da série de indicadores existentes, a utilização da Epidemiologia mostra-se como uma tarefa crítica aos gestores de SB do país. A escolha do indicador depende de quem os utilizará na tomada de decisão, de sua plausibilidade e do que se pretende avaliar: processo ou resultado com a implantação do serviço ${ }^{25}$. Para Tanaka e Tamaki ${ }^{26}$ a tomada de decisão deve ser subsidiada pelo processo avaliativo (retroalimentada em "círculo") e deve considerar as necessidades da população. Princípios como utilidade, oportunidade, factibilidade, confiabilidade, objetividade e direcionalidade constituem a estrutura para o estabelecimento de indicadores e parâmetros de avaliação para a gestão de serviços de saúde. Nesse contexto, ao pensar-se num critério para alocação de LRPD, o uso da média de dentes perdidos através do índice de utilização universal CPOD apresentar-se-ia como uma proposta de simples obtenção e baixo custo ${ }^{27}$. Por outro lado, não permitiria o monitoramento do impacto desses serviços sobre o indicador, que é irreversível. Uma alternativa seria o uso dos indicadores de necessidade de reposição protética ou o desenvolvimento de um índice que abrangesse múltiplas dimensões (e.g. necessidade normativas e expressadas-demanda). Além disso, como a condição social pode influenciar no padrão de utilização dos serviços de saúde ${ }^{28,29}$, o Índice de Desenvolvimento Humano (IDH), poderia ser agregado ao parâmetro ${ }^{30,31}$ para alocação desses recursos.

Neste estudo, as regiões com indicador epidemiológico ou de necessidade normativa mais elevada não foram aquelas com maior provisão de LRPD. Uma pesquisa realizada no Reino Unido $^{18}$ mostrou que $2 / 3$ dos inquéritos de avaliação de necessidade (e demanda) foram diretamente usados no processo de tomada de decisão. No entanto, $20 \%$ dos inquéritos realizados não tiveram impacto no planejamento dos serviços locais do sistema, sugerindo que a tomada de decisão, em algumas áreas, passaria por um pro- cesso de barganha ${ }^{18}$. No Brasil, a municipalização da saúde é apontada como possível justificativa para a dificuldade do gestor federal alocar recursos, uma vez que a solicitação depende da gestão municipal. Com a descentralização, os municípios passaram a ter maior autonomia ${ }^{32,33}$ para o desenvolvimento de programas locais de saúde e governabilidade sobre a adesão (ou não) aos programas federais. Por outro lado, em grande medida, as ações municipais dependem dos incentivos (financeiros) e controles estaduais e federais a que estão submetidos ${ }^{34}$. Desse modo, a gestão local pode utilizar diferentes critérios para tomada de decisão, de acordo com o modelo de governança municipal adotado ${ }^{35}$ e dos contratos de gestão pactuados ${ }^{36}$.

As diferenças regionais refletiram sobre as desigualdades de oferta e utilização dos LRPD. Compreende-se que o uso do serviço não depende unicamente da oferta do mesmo ou da necessidade clínica, visto que a autopercepção do indivíduo sobre sua saúde influi na demanda (procura) para serviços de saúde ${ }^{37}$. Todavia, as diferenças geográficas, econômicas, culturais e funcionais podem repercutir sobre as inequidades na utilização dos serviços, tanto entre países $^{29}$, quanto inter-regiões ${ }^{38}$. Neste estudo, isso foi percebido através da contradição entre a maior prevalência de edêntulos estar concentrada na região Sudeste, enquanto que a maior implantação de LRPD por habitante foi na região Nordeste. Já a maior necessidade reabilitadora de PT foi percebida na região Norte, mas a mesma apresentou a menor produção (utilização) entre as macrorregiões. Em contrapartida, as regiões com maior acesso (salvo para $\mathrm{PF}$ ) foram aquelas com maior provisão de LRPD, indo ao encontro de outros estudos com serviços de atenção bási$\mathrm{ca}^{39}$, de média complexidade ${ }^{30,40}$. Como também nos achados em relação aos equipamentos de $\mathrm{Rx}$ odontológicos ${ }^{41}$.

A produção média brasileira de próteses foi de 7,56 unidades $\backslash 100.000$ habitantes ao mês, o que pode ser compreendido como baixa, considerando o atual nível de necessidades clínicas. Entretanto, não há padrões comparativos de produtividade para se considerar a produção ambulatorial dos LRPD satisfatória (ou não). Alguns autores propuseram a discussão sobre a cobertura eficaz de um serviço de saúde, ou seja, aquele capaz de produzir o máximo de benefício para a população de acordo com suas necessidades ${ }^{42}$. Esse cálculo poderia ser empregado para se estimar a quantidade de LRPD necessários para cobrir a população edêntula diagnosticada e mi- 
nimizar as desigualdades regionais observadas. Atualmente, o monitoramento referente às próteses dentárias para repasse financeiro aos municípios ocorre de acordo com a faixa de produção/ mês pactuada. Desse modo, reitera-se a influência do acompanhamento dos LRPD, visto que a produção encontrada pode revelar problemas de gestão dos laboratórios, e não propriamente do município ${ }^{43}$.

O presente estudo é exploratório, apresentando limitações e pontos fortes. Não foi possível garantir a comparabilidade entre os indicadores epidemiológicos e as taxas de oferta e de utilização dos serviços, pois o denominador populacional foi diferente para distintos indicadores. A validade das informações de bases secundárias é ainda considerada discutível, todavia, seu uso tende a qualificar os registros em saúde e constitui uma vertente importante nos estudos avaliativos de serviços de saúde ${ }^{26}$. Para evitar vieses referentes a erros de notificação, foram utilizadas apenas grandes áreas de abrangência, em que tais viéses podem ser assumidos como aleatórios. Como aspecto positivo, este é um dos primeiros estudos sobre os LRPD. Até o momento, os estudos referentes à avaliação de serviços da atenção especializada em SB dão ênfase aos Centros de Especialidades Odontológicas ${ }^{40}$. Embora a evidência de que os serviços podem impactar na saúde seja pouca ${ }^{44}$, a garantia dos serviços (públicos) de saúde podem atuar na limitação de danos e recuperação de saúde de forma positiva nas condições de saúde bucal da população ${ }^{31}$. Os LRPD representam componentes de suma importância para a integralidade da atenção na rede pública de serviços de SB por abarcarem uma condição histórica e complexa como a perda dentária.

\section{Conclusão}

Os achados deste estudo sugerem que os aspectos para além da epidemiologia, como políticos, operacionais, administrativos, financeiros e de interesse do controle social podem ter influenciado na alocação de LRPD. Considerando-se a transitoriedade dos instrumentos de gestão e planejamento em saúde no âmbito municipal, o fomento de estratégias regionais de planejamento podem ser uma viável alternativa na tomada de decisão, distribuição desses recursos no SUS e na ampliação do acesso à população de municípios pequenos e de zonas rurais.

Acredita-se que as desigualdades regionais apresentadas podem ser minimizadas com a disponibilidade de recursos de estrutura e melhor organização dos serviços de saúde. Futuramente, seriam oportunas investigações sobre a implantação de LRPD no âmbito municipal e/ou estadual e que considerem aspectos contextuais, como, por exemplo, a cobertura dos demais serviços que compõem a rede de SB.

\section{Colaboradores}

VR Aguiar e RK Celeste participaram da concepção e desenho do estudo, revisão de literatura, análise de dados e interpretação. VRA redigiu o manuscrito e RKC revisou e aprovou a versão final. 


\section{Referências}

1. Institute for Health Metrics and Evaluation. The Global Burden of Disease: Generating Evidence, Guiding Policy. Washington: University of Washington; 2013.

2. Jovino-Silveira RC, Caldas Júnior AF, Souza EH, Gusmão ES. Primary reason for tooth extraction in a Brazilian adult population. Oral Health Prev Dent 2005; 3(3):151-157.

3. Murray TW. Epidemiology of oral health conditions in older people. Gerodontology 2013; 31(Supl. 1):9-16.

4. Emami E, de Souza RF, Kabawat M, Feine JS. The impact of edentulism on oral and general health. Int $J$ Dent 2013; 1(498305):7.

5. Fontanive V, Abegg C, Tsakos G, Oliveira M. The association between clinical oral health and general quality of life: a population-based study of individuals aged 50-74 in Southern Brazil. Community Dent Oral Epidemiol 2013; 41(2):154-162.

6. Gerritsen AE, Allen PF, Witter DJ, Bronkhorst EM, Creugers NH. Tooth loss and oral health-related quality of life: a systematic review and meta-analysis. Health Qual Life Outcomes 2010; 8:126.

7. Brasil. Ministério da Saúde (MS). Secretaria de Atenção à Saúde. Secretaria de Vigilância em Saúde. SB Brasil 2010 Pesquisa Nacional de Saúde Bucal. Resultados Principais. Brasília: MS; 2012.

8. Barbato PR, Muller Nagano HC, Zanchet FN, Boing AF, Peres MA. Tooth loss and associated socioeconomic, demographic, and dental-care factors in Brazilian adults: an analysis of the Brazilian Oral Health Survey, 2002-2003. Cad Saude Publica 2007; 23(8):1803-1814.

9. Pucca Júnior GA. The national politics of buccal health as social demand. Cien Saude Colet 2006; 11(1):243246.

10. Brasil. Ministério da Saúde (MS). Secretaria de Atenção à Saúde. Departamento de Atenção Básica. Coordenação Nacional de Saúde Bucal. Diretrizes da Política Nacional de Saúde Bucal. Brasília: MS; 2004.

11. Brasil. Portaria no 211 de 13 de maio de 2011. Secretaria de Atenção à Saúde. Ministério da Saúde. Altera os valores dos procedimentos da Tabela de Procedimentos, Medicamentos, Órteses, Próteses e Materiais Especiais do Sistema Único de Saúde (SUS) realizada pelos Laboratórios Regionais de Próteses Dentárias (LRPD), segundo critérios estabelecidos pela Política Nacional de Saúde Bucal. Diário Oficial da União 2011; 16 maio.

12. Brasil. Portaria no 2.374 de 7 de outubro de 2009. Gabinete do Ministro. Ministério da Saúde. Altera os valores dos procedimentos da Tabela de Procedimentos, Medicamentos, Órteses, Próteses e Materiais Especiais do Sistema Único de Saúde (SUS) realizada pelos Laboratórios Regionais de Próteses Dentárias (LRPD), segundo critérios estabelecidos pela Política Nacional de Saúde Bucal. Diário Oficial da União 2009; 9 out.
13. Brasil. Portaria no 1.825 de 24 de agosto de 2012. Altera o valor dos procedimentos de prótese dentária na Tabela de Procedimentos, Medicamentos e OPM do SUS e estabelece recursos anuais a serem incorporados ao Teto Financeiro Anual de Média e Alta Complexidade dos Estados, Municípios e Distrito Federal para confecção de próteses dentárias nos Laboratórios Regionais de Próteses Dentárias (LRPD). Diário Oficial da União 2012; 27 ago.

14. Brasil. Sala de Apoio à Gestão Estratégica. 2014 [cited 2014 May 20]. Available from: http://189.28.128.178/ sage/\#

15. Chaves SCL, Vieira da S. Oral health care and health decentralization in Brazil: two case studies in Bahia State. Cad Saude Publica 2007; 23(5):1119-1131.

16. Cohn A, Westphal MF, Elias PE. Data and the process of formulating health policies. Rev Saude Publica 2005; 39(1):114-121.

17. Manfredine M. Programação das ações em saúde bucal. Planejamento, gestão e avaliação em saúde bucal. São Paulo: Editora Artes Médicas; 2012.

18. Hensher M, Fulop N. The influence of health needs assessment on health care decision-making in London health authorities. J Health Serv Res Policy 1999; 4(2):90-95.

19. Roncalli AG. Epidemiology and public health dentistry: a shared walkway. Cien Saude Colet 2006; 11(1):105114.

20. Oliveira AGRC, Unfer B, Costa ICC, Arcieri RM, Guimarães LOC, Saliba NA. Epidemiological study in oral health: analysis of the methodology proposed by World Health Organization. Rev Bras Epidemiol 1998; 1(2):177-189.

21. Viacava F. Health information: the relevance of health surveys. Cien Saude Colet 2002; 7(4):607-621.

22. Brasil. Ministério da Saúde. Secretaria de Atenção à Saúde. Departamento de Atenção Básica. Projeto SB Brasil 2003: condições de saúde bucal da população brasileira 2002-2003: resultados principais. Brasília: MS; 2005. (Série C Projetos, Programas e Relatórios).

23. Vermelho LL, Costa AJL, Kale PL. Indicadores de Saúde. Epidemiologia. São Paulo: Editora Atheneu; 2002.

24. Brasil. Ministério da Saúde (MS). Departamento de Atenção Básica. Coordenação Geral de Saúde Bucal. Nota Técnica: Credenciamento e repasse de recursos para os Laboratórios Regionais de Próteses Dentárias - LRPD. Brasília: MS; 2012.

25. Habicht JP, Victora CG, Vaughan JP. Evaluation designs for adequacy, plausibility and probability of public health programme performance and impact. Int J Epidemiol 1999; 28(1):10-18.

26. Tanaka OY, Tamaki EM. The role of evaluation in decision-making in the management of health services. Cien Saude Colet 2012; 17(4):821-828. 
27. Guimarães LOC, Guimarães AMR. DMF Index simplification from 18 to 25 years. Rev Saude Publica 1990; 24(5):407-411.

28. Peres KG, Peres MA, Boing AF, Bertoldi AD, Bastos JL, Barros AJD. Reduction of social inequalities in utilization of dental care in Brazil from 1998 to 2008. Rev Saude Publica 2012; 46(2):250-258.

29. Celeste RK, Nadanovsky P, Fritzell J. Trends in socioeconomic disparities in the utilization of dental care in Brazil and Sweden. Scand J Public Health 2011; 39(6):640-648.

30. Scarpin JE, Slomski V. Estudo dos fatores condicionantes do índice de desenvolvimento humano nos municípios do estado do Paraná: instrumento de controladoria para a tomada de decisões na gestão governamental. Revista de Administração Pública 2007; 41(5):909-933.

31. Goes PSA, Figueiredo N, Neves JC, Silveira FMM, Costa JFR, Pucca Júnior GA, Rosales MS. Evaluation of secondary care in oral health: a study of specialty clinics in Brazil. Cad Saude Publica 2012; 28(Supl.):81-89.

32. Ugá MA, Francisco PS, Marta PS, Magalhães VS. Decentralization and resource allocation in the Brazilian National Health System (Sistema Único de Saúde SUS). Cien Saude Colet 2003; 8(2):417-437.

33. Sobrinho JEL, Espírito Santo ACG. Federal participation in the financing of primary oral healthcare, a study in Vitória de Santo Antão city, Pernambuco. Saúde e Sociedade 2013; 22(4):994-1000.

34. Arretche M. Federal financing and local management of social policies: the balance among regulation, accountability and autonomy. Cien Saude Colet 2003; $8(2): 331-345$

35. Fleury S, Ouverney ALM, Kronemberger TS, Zani FB. Local governance in the decentralized health care system in Brazil. Rev Panam Salud Publica 2010; 28(6):446-455.

36. Ditterich RG, Moysés SJ. A gestão da saúde baseada em resultados e processos de contratualização. Planejamento, gestão e avaliação em saúde bucal. São Paulo: Editora Artes Médicas; 2012.

37. Campos O. O estudo da demanda e das necessidade e sua importância para o planejamento em saúde. Rev Saude Publica 1969; 3:79-81.
38. Travassos C, Oliveira EXG, Viacava F. Geographic and social inequalities in the access to health services in Brazil: 1998 and 2003. Cien Saude Colet 2006; 11(4):975-986.

39. Fischer TK, Peres KG, Kupek E, Peres MA. Primary dental care indicators: association with socioeconomic status, dental care, water fluoridation and Family Health Program in Southern Brazil. Rev Bras Epidemiol 2010; 13(1):126-138.

40. Celeste RK, Moura FRRd, Santos CP, Tovo MF. Analysis of outpatient care in Brazilian municipalities with and without specialized dental clinics, 2010. Cad Saude Publica 2014; 30(3):511-521.

41. Freitas MB, Yoshimura EM. Survey on the distribution of imaging diagnosis equipment and frequency of radiological examinations in the State of São Paulo, Brazil. Radiologia Brasileira 2005; 38(5):347-354.

42. Shengelia B, Tandon A, Adams OB, Murray CJ. Access, utilization, quality, and effective coverage: an integrated conceptual framework and measurement strategy. Soc Sci Med 2005; 61(1):97-109.

43. Chaves SCL, Cruz DN, Barros SG, Figueiredo AL. Assessing the supply and use of secondary care in specialized dental clinics in Bahia State, Brazil. Cad Saude Publica 2011; 27(1):143-154.

44 Celeste RK, Nadanovsky P, Leon AP. Association Between Public Dental Service Preventive Procedures and Caries Prevalence. Rev Saude Publica 2007; 41(5):830838.

Artigo apresentado em 18/09/2014

Aprovado em 28/01/2015

Versão final apresentada em 30/01/2015 